\title{
Optimizing Consumption of Energy in Multicast model in -Constrained Mobile Wireless Networks
}

\author{
Yonas Melaku Waktola, Kokeb Abera
}

\begin{abstract}
This paper addresses the issue of saving energy consumption in a multicast model in mobile wireless networks with time limit, where source information must be sent to all destinations within an self imposed time limit. Many of the existing works focuses on getting transmission schemes with minimal energy transmission, bypassing the use of energy on the receiver side. Therefore, in this ConMap paper, a new and novel framework is proposed for an efficient design of the scheme employed in transmission that increases the efficiency of the transmission and reception energy together. Thus, the issue of designing a scheme of minimal energy transmission, DeMEM, will be given as one of combination in optimization and will show approximately ratio of polynomial time algorithm of DeMEM can not be better than (1/4) In $k$. In order to give very efficient approximation schemes, the proposed model ConMap converts DeMEM into an equivalent oriented direct shaft issue by creating helpdesk of graphic devices to capture the use of energy, maps the calculated tree into a scheme of transmission I

There are three advantages of ConMap:

1) General: ConMap shows a Clear application to a wide range of power models.

2) Flexibility:Any Steiner tree problem algorithm can be incorporated into our ConMap system to obtain spe cific guarantees and performance complexity.

Efficiency: The ConMap model retains the approximation ratio of the built-in Steiner tree algorithm, which will only tolerate a minor overload. These three features are empirically validated and in contrast with an exact brute force algorithm, ConMap also provides near-optimal transmission schemes. In this paper, energy transmission and reception are jointly considered in the design of multicast transmission schemes.
\end{abstract}

Keywords: Multicast, Mobile wireless networks, ConMap.

\section{INTRODUCTION}

Over recent years, the increase over mobile data traffic has dramatically increased the energy consumption in communications. As reported by a survey[1] that says Telecom Italia is Italy's second-largest consumer, the energy consumption of mobile communications already ranks first.

Therefore, energy saving becomes a crucial issue in the design and implementation of mobile wireless networks which receives considerable attention. Since most nodes operate with batteries, finding a route with minimum total energy consumption for a given communication session is a key problem in reducing the energy costs in mobile wireless networks[2].Because of the increasing demand for

Revised Manuscript Received on March 18, 2020.

Yonas MelakuWaktola, Department of Computer Science and Engineering, Jawaharlal Nehru Technological University, Hyderabad, India.

Kokeb Abera Workneh, Department of Computer Science and Engineering, Jawaharlal Nehru Technological University, Hyderabad, India. information exchange in these communication scenarios, multicast is more efficient in such circumstances.However, since a traffic model that generalizes both unicas $t$ and transmission, the problem of multicast minimum power is much more complicated than that of unicast minimal power, which is essentially a shorter route problem in static networks Of the existing studies that study the problem of minimal energy multicasting, most of them [3] are limited to static networks, which do not characterize many realistic scenarios in which users manifest themselves on the move. Consequently, the potential energy savings of mobility are also neglected. It is thus difficult to integrate the receiving energy directly into current algorithms. Which inspire us to introduce a general framework that optimizes the transmitting and the receiving of energy in mobile wireless networks together. This system, in addition to the resources, needs to incorporate the latency, another critical output metric of which demand differs in different scenarios. Following a theoretical analysis of the DeMEM problem's approach hardness, we are proposing a new framework for energy optimisation called ConMap. ConMap conducts multicast transmission in three main phases, in particular.

First, it creates an intermediate chart as a result of the chart status snapshots taken under D of different slots and auxiliary devices for transmitting and receiving energy. Second, build a Steiner tree on that intermediate card that covers all destinations. Finally, remap the tree obtained in that intermediate chart into a multicast transmission scheme in the original grid.

The ConMap proposal helps us to flexibly integrate any Steiner direct tree algorithm and it turns out that it $\mathrm{c}$ an be applied effectively with only a polynomial time complexity, as well as retaining the Steiner direct tree algorithm approach relationship. My structure is not based on any particular definition of energy consumption and is therefore applicable to a broad range of algorithms.

\section{PROBLEM STATEMENT}

Optimizing energy consumption in mobile wireless networks is a key problem. Improving energy use can he lp traffic control, save money and eventually improve lives.

In mobile wireless networks, low power consumption is a fundamental challenge, especially in sensor networks consisting of nodes considered light with limited battery charging. For mobile networks the most important mechanism is routing due to high energy consumption,

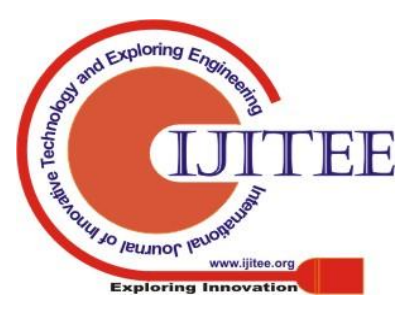


end-to-end latency and packet overload control. Therefore, a fast routing mechanism is needed to reduce the energy consumption.

Several routes can be used simultaneously to increase the routing efficiency of the wireless mobile networks. After processing, data is propagated in a consistent path, like duplicate prevention, timestamps, etc. Routing protocol efficiency is related to the architectural model, and depends largely on the implementation model. Restrictions on architecture could further affect performance[3 ]. The routing protocol is significantly influenced by its data delivery model, particularly with regard to the stability of the route and the minimization of energy consumption.

Following are some of the problems that mobile wireless networks face due to the lack of power optimization between the sender and the recipient

- Increased data traffic leading to high consumption of energy in communications

- Mobile communication is the main energy consumption.

- Most nodes run on battery power

- The potential mobility energy is neglected

- Optimal transmission side, without studying energy reception.

- Finding a Total energy consumption path minimum is a key problem

- Reception power: number of receivers, awaiting feedback, returning missing packets

- General framework necessary to jointly optimize the consumption of transmission and reception energy

\section{PROPOSED SYSTEM}

In this paper we introduce a new framework for the method: ConMap.Via theoretical analyzes and si mulations extended in actual network data sets, we have demonstrated the generality, versatility and efficien cy of ConMap system.

\section{A. Mobile wireless network model}

The mobile wireless network model[5 ] that can be replicated is followed as follows: time is divided into dis crete time intervals and the nodes remain static within a time interval. And this model allows a reasonable pr ediction of potential node movements. THe explanation for this is that node mobility and topology evolution largely depend on the social and temporal characteristics of the network's participants. A mobile network can be modelled as a series of static graphics with a fair prediction of future node movements:

$\mathrm{G}=\{\mathrm{G} 1, \mathrm{G} 2, \ldots\}$,For each static graph interpreted as a snapshot of the locations of nodes in a time slot. Referr ed specifically to as:

$\mathrm{t}(\mathrm{V}, \mathrm{Et}, \mathrm{w} \mathrm{t}) \in \mathrm{G}$, each static graph is a weighted directed graph with $\mathrm{V}$

$=\{\mathrm{v} 1, \mathrm{v} 2, \ldots, \mathrm{vn}\}$ corresponding to the $\mathrm{n}$ network nodes.

Et $\subseteq \mathrm{V} \times \mathrm{V}$ denotes the edge set and wt $: \mathrm{Et} \rightarrow \mathrm{R}+$ assigns each edge a corresponding weight. An edge $\mathrm{e}=(\mathrm{vi}, \mathrm{v} \mathrm{j}) \in$ Et means that vi can send messages to $\mathrm{vj}$ in time slot $\mathrm{t}$ with minimum transmission power wt(e).

\section{B. Multicast Model}

We consider a wireless multicast session with a source $s \in V$ and destination nodes set $\mathrm{T} \subseteq \mathrm{V}$ in mobile network $\mathrm{G}$. an integral delay constraint $\mathrm{D}$ on the multicast session is being imposed, in the sense that the message must be delivered to all nodes in $\mathrm{T}$ within $\mathrm{D}$ slots. Therefore, for a multicast session, we only need to consider the network snapshots within the delay constraint. Hence, in the sequel, we truncate the mobile networkG into D static graphs denoted as $\{\mathrm{G} 1, \mathrm{G} 2, \ldots, \mathrm{GD}\}$. Since we aim to design a minimum energy transmission scheme for a delay-constrained multicast session in a mobile wireless network, we next formally define the notion of transmission scheme. We refer to the process of some transmitter transmitting message to the intended receivers as a transmission. A transmission scheme specifies the transmitter, intended receivers, timing and transmission power of each transmission.

A transmission scheme $\pi \subseteq \mathrm{V} \times 2 \mathrm{~V} \times\{1, \ldots, \mathrm{D}\} \times \mathrm{R}+$ is a sequence of tuples $\{(\mathrm{u}, \mathrm{V}, \mathrm{t}, \mathrm{p})\}$, where a tuple $\tau=(\mathrm{u} \tau, \mathrm{V} \tau, \mathrm{t} \tau, \mathrm{p} \tau)$ $\in \pi$ denotes that node $u \tau$ should transmit to the nodes in $V \tau$ at time slot $t \tau$ with power $\mathrm{p} \tau$.

Also, in a transmission scheme $\pi$, we define a node to be "reached in time slot t" in a recursive way as follows: (i) the source $s$ is reached in time slot 1, (ii) if a node is reached in time slot $t$, then it is reached in time slot tfor all $t \geq t$, and (iii) if a node $\mathrm{u}$ is reached in time slot $\mathrm{t}$ and $(\mathrm{u}, \mathrm{V}, \mathrm{t}, \mathrm{p}) \in \pi)$, then all nodes in Vare reached after time slot $t$, or we say they have been reached in time slot $t+1$. Now, we give the definition of feasible transmission scheme and will restrict our consideration to the set of feasible transmission schemes in the sequel. Intuitively, a scheme is said to be feasible if it is without redundancy and qualified for the delay constrained multicast session.

\section{A Transmission Scheme II Is Feasible If It Observes The Following Conditions:}

(i) It ensures that $u$ is reached in $t$ and for all vs. V, e $=(u, v)$ $=(u, v)=E t$ and $p=\max e=(u, v) E t, v V\{w t(e)\}$ for all $\mu=(\mathrm{u}, \mathrm{V}, \mathrm{t}, \mathrm{p})$

(ii) No node in $\mathrm{V}$ is reached before $\mathrm{t}$ for all $\pi=(\mathrm{u}, \mathrm{V}, \mathrm{t}, \mathrm{p})$ adjacent to $t$.

(iii) All Tnodes are reached in slot D time. Figure 1 provides an example of a mobile wireless network, a s well as a feasible transmission scheme suggested in the network under a multicast session.

Transmission system The tuples refer to $\{(\mathrm{v} 1,\{\mathrm{v} 2, \mathrm{v} 4\}, 1, \mathrm{p} 1),(\mathrm{v} 2,\{\mathrm{v} 5\}, 2, \mathrm{p} 2),(\mathrm{v} 5,\{\mathrm{v} 6\} 4, \mathrm{p} 3)$.

\section{Energy Model}

For a stated transmission by tuple $\tau=(\mathrm{u} \tau, \mathrm{V} \tau, \mathrm{t} \tau, \mathrm{p} \tau)$

They model this transmission's energy consumption in the following way: $\mathrm{E}(\tau)=\mathrm{p} \tau+\mathrm{f}(|\mathrm{V} \tau|)$,

Where the first component denotes the energy consumed by the transmitter side and $\mathrm{f}$ is a function of the number of receivers intended to represent the energy receiving. Notice that the proposed structure is not limited to any particular $f$ and the choice of $f$ may be based on realistic settings [7],[9], it follows that the energy consumption of a $\pi$ transmission scheme is given by: $\mathrm{E}(\pi)=\mathrm{l}$ ' adjusted $\mathrm{E}(\ddot{\mathrm{y}})$.

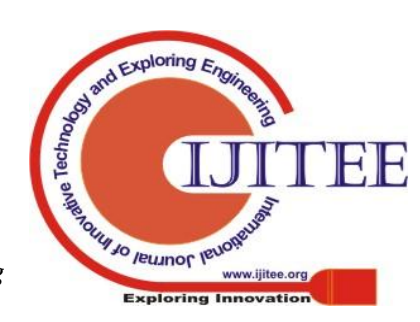




\section{SYSTEM DESIGN}

The DeMEM problem: Given a mobile wireless network $\mathrm{G}=$ $\{\mathrm{G} 1, \mathrm{G} 2, \ldots, \mathrm{GD}\}$, a source node s, a colle ction of destination nodes $\mathrm{T}$, and a delay constraint $\mathrm{D}$, the goal is to find a feasible transmission scheme with minimal energy usage.We remember that we make the following contact characterizations of mobile wireless networks in the descriptions above. Next, we assume the transmission rate is much higher than the mobility of the nodes. In other words, multiple transmissions into separate recipients in a single time slot are feasible. That may not be desirable if we only consider the cost of transmission. However, if we take the receiving energy into account it can bring potential gain. Second, we can use the wireless nature of broadcast during communications, i.e., different nodes can be reached within one transmission as long as the transmission power is sufficiently large. Finally, we concentrate in the present work on the multicasting of one data packet in the network, which relieves us from the burden of interruption and packet scheduling problems.

\section{The Mobile Network is Truncated into D Static Graphs $\{G 1, G 2, \ldots, G D\}$}

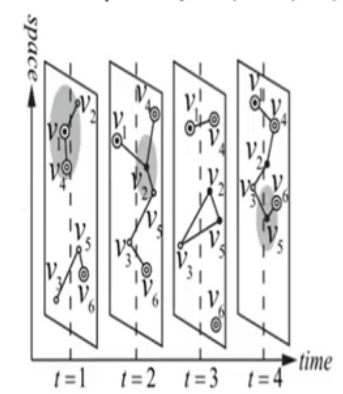

\section{$\{(v 1,\{v 2, v 4\}, 1, p 1),(v 2,\{(v 5\}, 2, p 2),(v 5,\{(v 6), 4, p 3)\}$}

tuple $\mathrm{r}=(\mathrm{ur}, \mathrm{V} \mathrm{t}, \mathrm{t} \tau, \mathrm{pr})$

$\mathcal{E}(\tau)=p_{\tau}+f\left(\left|V_{\tau}\right|\right)$

$\mathcal{E}(\pi)=\sum_{\tau \in \pi} \mathcal{E}(\tau)$

\section{CONCLUSION}

Finally the proposed system model ConMap increases the efficiency and consumes less energy

\section{REFERENCES}

1. Feng et al., "A survey of energy-efficient wireless communications," IEEE Commun.Surveys Tuts., vol. 15, no. 1, pp. 167-178, 1st Quart., 2013.

2. S. Guo and O. Yang, "Localized operations for distributed minimum energy multicast algorithm in mobile ad hoc networks," IEEE Trans. Parallel Distrib. Syst., vol. 18, no. 2, pp.186-198, Feb. 2007.

3. J. E. Wieselthier, G. D. Nguyen, and A. Ephremides, "Algorithms for energy-efficient multicasting in static ad hoc wireless networks," Mobile Netw. Appl., vol. 6, no. 3, pp. 251-263, Jun. 2001

4. P.-J. Wan, G. Calinescu, and C.-W. Yi, "Minimum-power multicast routing in static ad hoc wireless networks," IEEE/ACM Trans. Netw., vol. 12, no. 3, pp. 507-514, Jun. 2004.

5. W. L. Yang and L. J. Wang, "The investigation of delay-constrained multicasting with minimum-energy consumption in static ad hoc wireless networks," Int. J. Ad Hoc Ubiquitous Comput., vol. 4, nos. 3-4, pp. 237-250, Apr. 2009.

6. K. Han, Y. Liu, and J. Luo, "Duty-cycle-aware minimum-energy multicasting in wireless sensor networks," IEEE/ACM Trans. Netw., vol. 21, no. 3, pp. 910-923, Jun. 2013.

7. L. M. Feeney and M. Nilsson, "Investigating the energy consumption of a wireless network interface in an ad hoc networking environment," in Proc. IEEE INFOCOM, vol. 3. Apr. 2001,pp. 1548-1557.

8. N. A. Pantazis, S. A. Nikolidakis, and D. D. Vergados, "Energy-efficient routing protocols in wireless sensor networks: A survey," IEEE Commun. Surveys Tuts., vol. 15, no. 2, pp. 551-591, 2nd Quart., 2013.
9. M. P. Johnson, B. Phelan, A. Bar-Noy, P. Basu, and R. Ramanathan, "Minimum-cost network-wide broadcast over reliable MAC-layer multicast," IEEE Trans. Mobile Comput., vol.16, no. 12, pp. 3390-3402, Dec. 2017.

10. D. Jiang, Z. Xu, W. Li, and Z. Chen, "Network coding-based energyefficient multicast routing algorithm for multi-hop wireless networks," J. Syst. Softw., vol. 104, pp. 152-165, Jun. 2015.

11. X. Kang, C. K. Ho, and S. Sun, "Full-duplex wireless-powered communication network with energy causality," IEEE Trans. Wireless Commun., vol. 14, no. 10, pp. 5539-5551, Oct.2015.

12. T. Small and Z. J. Haas, "Resource and performance tradeoffs in delay-tolerant wireless networks," in Proc. ACM SIGCOMM Workshop Delay-Tolerant Netw., Philadelphia, PA, USA, Aug. 2005, pp. 260-267.

13. M.-R. Ra et al., "Energy-delay tradeoffs in smartphone applications," in Proc. ACM MobiSys, San Francisco, CA, USA, Jun. 2010, pp. 255-270.

14. S. Cui, R. Madan, A. Goldsmith, and S. Lall, "Energy-delay tradeoffs for data collection in TDMA- based sensor networks," in Proc. IEEE ICC, Seoul, South Korea, May 2005, pp. 3278-3284.

15. M. 'Cagal, J.-P. Hubaux, and C. Enz, "Minimum-energy broadcast in allwireless networks: NP- completeness and distribution issues," in Proc. ACM MobiCom, Atlanta, GA, USA, Sep.2002, pp. 172-182.

\section{AUTHORS PROFILE}

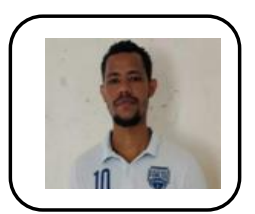

Yonas MelakuWaktola receivedB.Sc degree from Wolkite University, Ethiopia in Information Systems 2016. Working in Department of Information Systems, Mizan Tepi University, Ehiopia as an Assistant Lecturer. Currently pursuing Master's degree with the department of computer science and Engineering Jawaharlal Nehru Technological University Hyderabad, India in Cyber Forensics and Information Security.

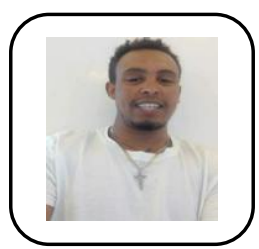

Kokeb Abera Workneh received B.Sc degree from Walaita Sodo University, Ethiopia in Computer Science 2016. Working in Department of Computer Science, Mizan Tepi University, Ethiopia as Assistant Lecturer. Currently pursuing Master's degree with the department of computer science and Engineering Jawaharlal Nehru Technological University Hyderabad, India in Cyber Forensics and Information Security. 